\title{
Therapeutic Potentials and Applications of Folic Acid and Beta Carotene
}

\author{
Bharat Kwatra $^{1^{*}}$, Invenzion labs \\ Rachit Modi², B. S. Anangpuria Institute of Pharmacy, Delhi-NCR, India
}

\author{
Article Info \\ Volume 7, Issue 4 \\ Page Number: 271-282 \\ Publication Issue : \\ July-August-2020
}

\section{Article History}

Accepted : 15 Aug 2020

Published : 22 Aug 2020

\section{ABSTRACT}

The present review is based mainly on papers published between 2000 and 2020 and gives information about the properties of the Folic Acid and Beta Carotene in chemical and biological systems and its possible role in preventing several diseases. The main aim of this report is to highlight its role as an immunopathological applications, also reported are bioactive properties that may influence the development of foam cells and protection against endothelial cell damage.

Keywords: Therapeutic, Bioactive, Folic Acid, Folate Deficiency

\section{Therapeutic Application of Folic Acid}

Folic acid is the synthetic form of folate, which is a naturally occurring $\mathrm{B}$ vitamin. Folate helps make DNA and other genetic material. It is especially important in prenatal health. Folate, also called vitamin $B-9$, is a $B$ vitamin that naturally occurs in certain foods. Folate is found in a number of plant and animal foods, including spinach, kale, broccoli, avocado, citrus fruits, eggs, and beef liver.

Your body uses folate for a wide array of critical functions, including the synthesis, repair, and methylation - the addition of a methyl group - of DNA. Also it is used incellular division. Used in Conversion of homocysteine to methionine, an amino acid that's used for protein synthesis or converted into S-adenosylmethionine (SAMe), a compound that acts as a primary methyl donor in your body and is necessary for numerous cellular reactions and in maturation of RBC.
Folate deficiency and folic acid supplementation: the prevention of neural-tube defects and congenital heart defects.

- Diet, particularly vitamin deficiency, is associated with the risk of birth defects.

- Characteristics of common and severe neuraltube defects together with congenital heart defects (CHD) as vitamin deficiencies play a role in their origin.

- The findings of the Hungarian intervention (randomized double-blind and cohort controlled) trials indicated that periconceptional folic acid (FA)-containing multivitamin supplementation prevented the major proportion (about 90\%) of neural-tube defects (NTD) as well as a certain proportion (about $40 \%$ ) of congenital heart defects. 
The Interest of Folic Acid in Targeted Photodynamic Therapy.

- Photodynamic therapy is an alternative to chemotherapy and radiotherapy for cancer treatment. PDT is clinically applied to treat age-related macular degeneration and several types of cancer.

- $\quad$ The use of folic acid as a promising targeting unit to improve the selectivity of photosensitizers to folic acid receptors.

- Strategies that are developed to couple folic acid to photosensitizers or nanoparticles as well as the influence of the presence of folic acid on the objects' photophysical properties and their effects on selectivity and phototoxicity in vitro and in vivo.

Effects of Folic Acid on DNMT1, GAP43, and VEGFR1 in Intrauterine Growth Restriction Filial Rats.

- To investigate the effect of a folic acid intervention on the outcome of intrauterine growth restriction (IUGR) filial rats.

- Changes in DNA methyltransferase 1 (DNMT1), growth-associated protein 43 (GAP43), and vascular endothelial growth factor receptor 1 (VEGFR1).

Folic acid conjugated nanoliposomes as promising carriers for targeted delivery of bleomycin.

- Targeted drug delivery has received considerable attention due to its key role in improving therapeutic efficacy and reducing the side effects of anticancer drugs.

- Bleomycin (BLM) is an anticancer antibiotic with short half-life, low therapeutic and high side effects that limit its clinical applications.
- $\quad$ Evaluation of the anticancer potential of folatetargeted liposomal bleomycin (FL-BLM) and its free-folate form (L-BLM) on two different cancer cell lines including human cervix carcinoma HeLa, and human breast carcinoma MCF-7 cells.

The effect of FL-BLM in induction of apoptosis and cell cycle arrest.

\section{Y-shaped Folic Acid-Conjugated PEG-PCL Copolymeric Micelles for Delivery of Curcumin.}

- Curcumin is a natural hydrophobic product showing anticancer activity and many studies show its potential use in the field of cancer treatment due to its safety and efficiency.

- Its application is limited due to its low watersolubility and poor selective delivery to cancer.

A Y-shaped folic acid-modified poly (ethylene glycol)-b-poly ( $\varepsilon$-caprolactone) 2 copolymer was prepared to improve curcumin solubility and realize its selective delivery to cancer.

Stable Liposome in Cosmetic Platforms for Transdermal Folic acid delivery for fortification and treatment of micronutrient deficiencies.

Oral folate fortification has been successful in many developed nations, however, developing countries still face low compliance and high incidence of folate deficiency associated with low birth weight infants and preterm deliveries.

- Safe and efficient approach for transdermal systemic folate delivery using fluidising liposomes $(120 \pm 4 \mathrm{~nm})$ stabilised within 3D matrix of naturally occurring cosmetic bases: Fuller's earth and henna with room temperature stability.

- The platform technology opens the possibility of delivering multiple nutrients systemically 
through skin and can be scaled for affordable community fortification.

Folic Acid-Targeted Etoposide Cubosomes for Theragnostic Application of Cancer Cell Imaging and Therapy.

- $\quad$ Study was to develop a novel Poloxamer-based drug delivery system featuring a tumortargeting folate moiety, which was expected to provide better targeting properties and therapeutic effects compared with the traditional cubosomes (Cubs).

- $\quad$ The folate-modified cubosomes containing ETP may be a promising drug candidate for antitumor treatment.

Preparation of Folic Acid-Targeted TemperatureSensitive Magnetoliposomes and their Antitumor Effects In Vitro and In Vivo

- Ovarian cancer is a common gynecologic malignancy with poor prognosis, requiring innovative new therapeutic strategies.

- $\quad$ Temperature-controlled drug delivery to cancer cells represents a novel, promising, targeted treatment approach.

- Folic acid-conjugated 17-AAG magnetic thermosensitive liposomes in combination with an alternating magnetic field for heating can achieve a synergistic anti-tumor effect of chemotherapy and heat treatment, potentially offering a new method for ovarian cancer treatment.

The antitumor effect of folic acid conjugatedAuricularia auricular polysaccharide-cisplatin complex on cervical carcinoma cells in nude mice.

- Tumor-targeted, folic acid (FA) conjugatedAuricularia auricular polysaccharide (AAP) - cis-diaminedichloroplatinum (CDDP) complex (FA-AAP-CDDP) was used for cervical carcinoma chemotherapy.

- $\quad$ The drug delivery system was able to enhance the antitumor potency of CDDP, and to reduce the toxic side effects of CDDP.

- New direction for folate receptor targeted polymers to improve anti-tumor activity but reduce side effects of CDDP.

Targeting the Folate Receptor: Improving Efficacy in Inorganic Medicinal Chemistry.

- The discovery of the high-affinity, highspecificity folate receptor in mamalian kidney cells, coupled with the ability of folate to enter cells by folate receptor-mediated endocytosis and the subsequent elucidation of the folate receptor's overexpression in specific cancer cell types; heralded the arrival of the area of chemotherapeutic folate targeting.

- The application of purely organic folate-based small-molecule drug conjugates that selectively target the folate receptor, which is over expressed in several diseases such as cancer, is well established.

- The application of inorganic folate-targeted drugs offers significant potential to expand and enhance this therapeutic approach.

- The union of folate-receptor targeting, and inorganic medicine may also lead to the development of treatments for disorders such as chronic-inflammation, tuberculosis, neurodegenerative disease and leishmaniasis.

Role of generation on folic acid-modified poly(amidoamine) dendrimers for targeted delivery of baicalin to cancer cells.

Baicalin (BAI) has been reported to exert antitumor effects. 
- However, BAI has limited water solubility, non-specific tumor targeting, and low bioavailability, which severely limited its clinical application.

- It was suggested that the PAMAM-FA have the potential for targeted delivery of BAI into cancer cells to enhance its anti-tumor efficacy.

Folic acid conjugated Fe 3 O 4 magnetic nanoparticles for targeted delivery of doxorubicin.

- The interfacial engineering of magnetic nanoparticles (MNPs) with specific functional groups or targeting ligands is important for their in vivo applications.

- Preparation and characterization of bifunctional magnetic nanoparticles (BMNPs) which contain a carboxylic moiety for drug binding and an amine moiety for folate mediated drug targeting.

- Nanoparticles also show high loading affinity for an anticancer drug, doxorubicin hydrochloride (DOX) and its $\mathrm{pH}$ dependent release. This is highly beneficial for cancer therapy as the relatively low $\mathrm{pH}$ in tumors will specifically stimulate the drug release at the site of interest.

- $\quad$ Fluorescence microscopy and flow cytometry studies confirmed the higher cellular internalization capability of these folic acid conjugated nanoparticles in cancer cells overexpressing folate receptors.

Non-covalent Nanocomplexes of Folic Acid and Reducible Polyethylenimine for Survivin siRNA Delivery.

- Efficient delivery of siRNA is critical for its therapeutic applications.

- Designing, synthesizing and evaluation of a novel delivery system based on non-covalent complexes of folic acid (FA) and a reducible polyethylenimine (PEI) derivative, PEI-SS.

SS is a potent siRNA carrier that warrants further evaluation.

Folic acid and creatine improve the firmness of human skin in vivo.

- The decrease in firmness is a hallmark of skin aging. Accelerated by chronic sun exposure, fundamental changes occur within the dermal extracellular matrix over the years, mainly impairing the collagenous network.

- Qualitative and quantitative assessment of skin firmness, in vitro and in vivo studies were carried out to elucidate the effects of topical folic acid and creatine to counteract this agedependent reduction in the amount of collagen. Treatment of fibroblast-populated dermal equivalents with folic acid and creatine increased collagen gene expression and procollagen levels and improved collagen fiber density, suggesting that the in vivo effects are based on the overall improvement of the collagen metabolism.

Folic Acid Promotes Recycling of Tetrahydrobiopterin and Protects Against HypoxiaInduced Pulmonary Hypertension by Recoupling Endothelial Nitric Oxide Synthase.

- $\quad$ Nitric oxide (NO) derived from endothelial NO synthase (eNOS) has been implicated in the adaptive response to hypoxia.

An imbalance between 5,6,7,8tetrahydrobiopterin (BH4) and 7,8dihydrobiopterin (BH2) can result in eNOS uncoupling and the generation of superoxide instead of NO.

Dihydrofolate reductase (DHFR) can recycle $\mathrm{BH} 2$ to $\mathrm{BH} 4$, leading to eNOS recoupling. 
Folic acid targeted $\mathrm{Mn}: \mathrm{ZnS}$ quantum dots for theranostic applications of cancer cell imaging and therapy.

- Synthesized a multifunctional nanoparticulate system with specific targeting, imaging, and drug delivering functionalities.

- Functionalization of the chitosan-encapsulated QDs with folic acid enhanced the internalization and binding affinity of the nanocarrier toward folate receptoroverexpressed cells.

- Due to the nontoxic nature of the composite, the as-synthesized nanoparticulate system can be used as a promising candidate for the ranostic applications, especially for a simultaneous targeted drug delivery and cellular imaging.

Folic acid enhances early functional recovery in a piglet model of pediatric head injury.

- $\quad$ For stroke and spinal cord injury, folic acid supplementation has been shown to enhance neurodevelopment and to provide neuroprotection.

- Hypothesized that folic acid would reduce brain injury and improve neurological outcome in a neonatal piglet model of traumatic brain injury (TBI).

Folic acid may enhance the early functional recovery in this piglet model of pediatric head injury.

- Development and characterization of folic acidconjugated chitosan nanoparticles for targeted and controlled delivery of gemcitabinein lung cancer therapeutics.

- Investigating the tumor-targeting potential of gemcitabine (GEM)-loaded surface-tailored chitosan (CS)/poly (ethylene glycol) nanoparticles (FA-PEG-GEM-NPs).

Interpreted that surface-tailored nanoparticles are capable to ferry bioactives selectively and specifically to tumor sites with the interception of minimal side effects, thereby suggesting their potential application in cancer therapeutics.

\section{Clinical trials on folic acid}

High-Dose Folic Acid in Preventing Colorectal Cancer in Patients Who Have Had Polyps Surgically Removed.

Trial id: NCT00002650

Patients are stratified according to participating center and age (50-70 vs 30-49 and over 70). Patients with adenomatous polyps at least $10 \mathrm{~mm}$ at colonoscopy are randomized to 1 of 2 treatment arms. Arm I: Patients receive folic acid daily. Arm II: Patients receive placebo daily. Treatment continues in both arms for 1 year in the absence of unacceptable toxicity or disease progression.

Study of Pralatrexate With Vitamin B12 and Folic Acid Supplementation for Previously Treated Recurrent or Metastatic Head and Neck Squamous Cell Cancer (HNSCC).

Trial id: NCT01183065

Patients were treated with $30 \mathrm{mg} / \mathrm{m} 2$ of pralatrexate intravenously once weekly for 3 weeks in a 4 week cycle with vitamin supplementation. Patients will take 1.0-1.25 mg oral folic acid on a daily basis. Folic acid should be initiated during the 10-day period preceding the first dose of pralatrexate and dosing will be continued during the full course of therapy and for 30 days after the last dose of pralatrexate. Patients will also receive a vitamin B12 (1 mg) intramuscular injection no more than 10 weeks prior 
to the first dose of pralatrexate and every 8-10 weeks thereafter. Subsequent vitamin B12 injections may be given the same day as treatment with pralatrexate.

\section{Radiolabeled Folic Acid and Imaging to Detect Ovarian Cancer.}

Trial id: NCT00003763

Patients receive an injection of folic acid conjugated with indium In 111. The patient then undergoes imaging studies at various time points. Patients then undergo exploratory surgery, the results of which are then compared to the imaging studies.

Folate-Depleted Diet Compared With FolateSupplemented Diet in Preventing Colorectal Cancer in Patients at High Risk for Colorectal Cancer.

Trial id: NCT00096330

Patients are placed on an average folate-containing diet for 56 days. After completion of the run-in period, patients are randomized to 1 of 2 arms. In arm 1 Patients are placed on a low-folate diet for 84 days. Patients receive oral folic acid supplementation once daily on days 57-84. In arm 2 Patients continue on an average folate-containing diet for an additional 56 days. Patients receive oral folic acid supplementation once daily on days 1-56.

Phase Ib Trial of Two Folate Binding Protein Peptide Vaccines (E39 and J65) in Breast and Ovarian Cancer Patients (J65)

Trial id: NCT02019524

$500 \mathrm{mcg}$ of lyophilized E39 peptide is suspended in bacteriostatic saline for injection and then frozen. At the time of vaccine administration, one vial of frozen suspended peptide is thawed and mixed with $250 \mathrm{mcg}$ GM-CSF in the syringe. This constitutes the E39 peptide vaccine. For patients randomized to the E39 vaccine arm, the primary vaccine series (PVS) consists of E39 vaccine administered intradermally every three to four weeks for six total vaccinations. After completion of the PVS, patients are assessed for significant residual immunity (SRI). Patients with SRI will be randomized to receive one inoculation of either the E39 vaccine or the J65 vaccine. Patients without SRI will be randomized to receive one inoculation of either the E39 vaccine or the J65 vaccine.

\section{A Study for Patients With Non-Squamous Non-Small Cell Lung Cancer. \\ Trial id: NCT00609518}

$500 \mathrm{mg} / \mathrm{m}^{\wedge} 2$ intravenous infusion on day 1 of each 21-day cycle. Number of Cycles: Until progression or to a maximum of 6 cycles.

Folic acid 350-1000 micrograms taken orally for at least 5 daily doses during the 7-day period prior to the first dose of pemetrexed then continues daily throughout treatment until 3 weeks after the last dose of pemetrexed.

\section{Folic Acid Clinical Trial for the Prevention of Cervical Cancer.}

Trial id: NCT00703196

Patients are stratified according to multivitamin use (yes vs no) and smoking status (smoker vs nonsmoker) Patients are randomized to 1 of 2 treatment arms. In arm 1 patients receive oral folic acid pill once daily for 12 months in the absence of unacceptable toxicity or any other adverse effects. In arm 2 patients receive oral placebo once daily for 12 months in the absence of unacceptable toxicity or any other adverse effects.

Multi-micronutrient Supplementation During Periconception and Congenital Heart Disease.

Trial id: NCT02537392 
Dietary Supplement: Vitamin B Complex and Folic Acid

Daily oral dose containing $2 \mathrm{mg}$ vitamin B1, $2 \mathrm{mg}$ vitamin B2, $2 \mathrm{mg}$ vitamin B6, $2 \mu \mathrm{g}$ vitamin B12, $2 \mathrm{mg}$ calcium pantothenate, $15 \mathrm{mg}$ nicotinamide and 0.4 mg folic acid.

\section{Therapeutic Application of $\beta$-carotene}

\section{INTRODUCTION}

$\beta$-Carotene is an organic, strongly coloured redorange pigment abundant in fungi, plants, and fruits. It is a member of the carotenes, which are terpenoids, synthesized biochemically from eight isoprene units and thus having 40 carbons.

$\beta$-carotene in Obesity Research: Technical consideration and current status of the field.

- Over the past decades, obesity has become a rising health problem due to accessibility to high calories, low nutrients value in the food.

- Bio-active components like $\beta$-carotene can contribute in the prevention and treatment of obesity.

- $\quad \beta$-carotene decreases obesity by promoting fatty acid oxidation in adipocytes and other tissues.

\section{$\beta$-carotene accumulation in serum and skin.}

- A single 51-mg dose of $\beta$-carotene given in the absence of dietary fibre result into no detectable change in the serum $\beta$-carotene. The same dose administered with $200 \mathrm{~g}$ fat increased serum $\beta$ carotene 2.5 fold at $40 \mathrm{~h}$.

- $\quad$ Similarly, administering $\beta$-carotene daily three divided doses with meals raised the serum $\beta$ carotene concentration three times as high compared with the same total dose administered once a day.
- $\quad$ These data indicate that $\beta$-carotene absorption requires dietary fats and is enhanced by administering with meals but there is a long time constant for serum (10 d) and tissue (several weeks) accumulation.

Improved absorption of $\beta$-carotene by encapsulation in an oil-in-water nanoemulsion tea polyphenols in the aqueous phase.

- $\quad \beta$-carotene serves as an important source of provitamin A and natural edible pigment, but the application is limited because of its instability and low oral-bioavaibility.

- A tea polyphenols- $\beta$-carotene oil-in-water nanoemulsion was prepared with the core oil phase containing $\beta$-carotene and water phase containing tea-polyphenols.

- Results suggest that tea polyphenols are effective ingredients for improving the oral-bioavailability of $\beta$-carotene.

$\beta$-carotene therapy for erythropoetic protoporphyria and other photosensitive disease.

- $\quad \beta$-carotenoid pigments can be used in the treatment of light-sensitive skin disease.

- The possible mechanism of carotenoid photoprotective and anti-cancer actions were mentioned.

$\beta$-carotene and the application of transcriptomics in risk-benefit evaluation of natural dietary components.

- $\quad \beta$-carotene is an anti-oxidant and is a source of vitamin- A.

- It is endowed with health beneficial properties, but high intake of $\beta$-carotene can lead to lung cancer. 
$\beta$-carotene loaded zein nanoparticles to improve the biopharmaceutical and to abolish the toxicity of methotrexate: a preclinical study of breast cancer.

- $\quad \beta$-carotene loaded nanoparticles of zein were developed using modified phase separation technique.

- The combined effect of $\beta$-carotene and its nanoparticles counterpart with methotrexate was evaluated thereafter for cytotoxicity and apoptotic activity in MCF-7 cells.

- Zein nanoparticles improve the cellular uptake, cytotoxicity and exhibited enhanced oralbiopharmaceutical and performance of $\beta$ carotene.

- This combination regimen could also be promising platform to facilitate the therapeutic benefits of anti-cancer agents.

\section{$\beta$-carotene-loaded Nanostructured Lipid Carriers.}

- Nanostructured lipid carriers (NLC) technology was used to dispense hydrophobic $\beta$-carotene in an aqueous phase.

- $\quad$ Stability of the $\beta$-carotene in water depends on NLC concentration and tocophenol content was measured photospectrometrically to get an estimation of the behaviour of NLC in beverages.

Microemulsions as delivery systems of lemon oil and $\beta$-carotene into beverages: Stability test under different light conditions.

- The stability of a model beverage containing a microemulsion delivering $\beta$-carotene and lemon oil was tested under increasing light intensity upto $6000 \mathrm{~lx}$ at $20^{\circ} \mathrm{C}$.

- Transparent microemulsions can be used to deliver flavoured oil and colourants into beverages. However, the photostability of the delivered compounds should be studied to estimate product shelf life.

\section{CLINICAL TRAILS ON $\beta$-CAROTENE}

\section{$\beta$-CAROTENE TRIALS ON MATERNAL AND INFANT MORTALITY IN BANGLADESH}

Impact of Maternal Vitamin A or BetaCarotene Supplementation on Maternal and Infant Mortality in Bangladesh

\section{Trial ID: NCT00198822}

The purpose of this trial is to determine whether providing women with a weekly oral supplement of vitamin $\mathrm{A}$, either preformed or as beta-carotene, at a dosage equivalent to a recommended intake from early pregnancy through three months postpartum, can reduce the risk of maternal mortality, fetal loss, or infant mortality. Women were recruited into the trial from August 2001 to January 2007. As this was a community trial, we identified pregnant women by conducting a urine test on women reporting to be amenstrual from the last visit during 5 weekly visit cycles to their homes in rural communities of the districts of Rangpur and Gaibandha, Bangladesh.

\section{NOISE-INDUCED HEARING LOSS TRIALS}

Micronutrients to Prevent Noise-induced Hearing Loss

\section{Trial ID: NCT00808470}

Noise-induced hearing loss (NIHL) is a significant clinical, social, and economic issue. Studies in animals have allowed us to identify mechanisms contributing to NIHL, including direct mechanical trauma, free radicals formed in association with metabolic stress, and reduced blood flow. A combination of antioxidant vitamins (beta-carotene, and vitamins $\mathrm{C}$ and $\mathrm{E}$ ) and the mineral magnesium (which acts in part as a vasodilator but also as an antioxidant) is highly effective in preventing NIHL in animals. These 
studies evaluate efficacy of this intervention in humans.

Hypothesis: Treatment with these micronutrients provides safe, effective attenuation of acute hearing changes induced by exposure to real-world sounds producing temporary (non-permanent) or permanent hearing changes induced by exposure to real-world sounds.

\section{TRIAL ON FVJC}

Fruit and Vegetable Juice Concentrate Effects on RBP4 and Antioxidant Capacity in Lean and Overweight 6-10 Year Old Boys (FVJC) Trial ID: NCT00842543

The study aims to evaluate if the consumption of a Fruit and Vegetable Juice Concentrate (FVJC) vs. placebo can exert changes in the serum levels of Retinol Binding Protein 4 (RBP4), carotenoids, tocophenol, antioxidant capacity, pro-inflammatory markers, and insulin sensitivity in conjunction with weight management in a cohort of lean as compared to overweight healthy six to ten year-old boys. The boys will undergo a detailed medical and family history and a complete physical examination during the screening visit. Careful anthropometric measures and body composition by DEXA will be performed. Nutritional and exercise counseling will be given at the start of the study. The outcome measures will include differences in serum RBP4, antioxidant levels, inflammatory markers and insulin sensitivity at baseline and six months after consumption of FVJC in a randomized double blind placebo control manner.

TRIALS ON ANTIOXIDANT-ERICHED MULTIVITAMIN SUPPLEMENT

Effects of an Antioxidant-Enriched Multivitamin Supplement on Inflammation and Oxidative Stress in Cystic Fibrosis (AquADEKs-2)

Trial ID: NCT01859390
The purpose of this study will be to evaluate the effects of a modified formulation of AquADEKs (AquADEKs-2) on markers of inflammation, antioxidant levels and oxidative stress.

Cystic Fibrosis (CF) is a disease that affects the organs in the body such as the lungs. Some of the damage to the lungs of CF patients may be caused by something called oxidant/antioxidant imbalance and oxidative stress.

Oxidation in the body is kind of what happens to an apple when it turns brown after being cut. And, just as a squeeze of lemon juice stops the oxidation of an apple, antioxidants can stop the rusting (or damage) inside our bodies by unstable oxygen molecules called free radicals. Free radicals can help fight off bacteria and viruses but too many of them do damage instead. Our bodies need antioxidants to keep things in balance so we have the right amount of free radicals.

Many CF patients also have trouble digesting food and absorbing nutrients like vitamins. Many of the vitamins we rely on are antioxidants, like vitamins $\mathrm{A}$, $\mathrm{D}, \mathrm{E}, \mathrm{K}$ and beta-carotene. In some people with $\mathrm{CF}$, even though they take multivitamins and pancreatic enzymes, they still have low amounts of antioxidants. The investigators are looking to see if taking more vitamins and antioxidants will help CF patients. AquADEKs-2 is an investigational new drug (a drug that has not received approval by the Food and Drug Administration [FDA]). This research study is being done with the AquADEKs-2 compared to a control multivitamin. The study drug, AquADEKs-2 contains standard amounts of fat-soluble vitamins (A, D, E, K) that are contained in typical CF multivitamin supplements plus several antioxidants including betacarotene, mixed tocophenol (different forms of vitamin $\mathrm{E})$, coenzyme Q10 (CoQ10), mixed carotenoids (lutein, lycopene and zeaxanthin), and the minerals zinc and selenium. The control multivitamin contains standard amounts of vitamins $\mathrm{A}, \mathrm{B}, \mathrm{D}, \mathrm{E}$, and $\mathrm{K}$ without additional antioxidant supplementation. 


\section{CLINICAL TRIAL ON AGE-RELATED EYE} DISEASE

\section{Age-Related Eye Disease Study 2 (AREDS2) (AREDS2) Trial ID: NCT00345176}

Oral supplementation with the Age-Related Eye Disease Study (AREDS) formulation (antioxidant vitamins $\mathrm{C}$ and $\mathrm{E}$, beta carotene, and zinc) has been shown to reduce the risk of progression to advanced age-related macular degeneration (AMD). Observational data suggest that increased dietary intake of lutein + zeaxanthin (carotenoids), omega-3 long-chain polyunsaturated fatty acids (docosahexaenoic acid [DHA] + eicosapentaenoic acid [EPA]), or both might further reduce this risk. AREDS2 was designed to test whether adding lutein + zeaxanthin, DHA + EPA, or lutein + zeaxanthin and DHA + EPA to the AREDS formulation might further reduce the risk of progression to advanced AMD. A secondary goal was to test the effects of eliminating beta carotene and reducing zinc dose in the AREDS formulation.

\section{CLINCIAL TRIAL OF OMEGA ON MALIGNANCY} Comparison of Carotenoid Bioavailability from Fresh

\section{Papaya, Tomato and Carrot}

Trial ID: NCT01748916

The goal of this study is to determine if papaya fruits are an exceptionally good food source for carotenoids in humans, particularly when compared more common carotenoid sources like carrots and tomatoes. This objective will be accomplished by quantitation of the immediate postprandial plasma concentrations of parent carotenoids and vitamin A metabolites from subjects who consumed a meal containing fresh papaya, tomato, and carrot.
TRAILS OF GLUTAMINE AND ANTIOXIDANT SUPPLEMENT

Trial of Glutamine and Antioxidant Supplementation in Critically Ill Patients (REDOXS)

Trial ID: NCT00133978

Critically ill patients experience a degree of hyper inflammation, cellular immune dysfunction, and oxidative stress. Supplementation with key nutrients, like glutamine and antioxidants, is most likely to have a favourable effect on these physiological parameters leading to an improvement in clinical outcomes. The results of two separate meta-analyses suggested that glutamine and antioxidants may be associated with improved survival. The purpose of this study is to determine whether providing high dose glutamine and antioxidants to critically ill patients will be associated with improved survival.

\section{TRAILS ON LUTEIN SUPPLEMENT}

Effects of Lutein Supplementation on Subclinical Atherosclerosis

\section{Trial ID: NCT01534533}

Atherosclerosis is the primary cause of cardiovascular and cerebrovascular diseases, both of which are the top two causes of death in industrialized countries including China. Lutein was found to be protective against atherosclerosis in some case control studies. However, the intervention on atherosclerosis has not been reported. In the present study, 192 subjects were randomly assigned to four groups treated with different amounts of lutein. The investigators observe the changes of serum lutein concentration by hyperpressure liquid chromatography (HPLC), and compare the differences of common carotid IMT and arterial stiffness by carotid ultrasonography before and after the intervention. Serum biochemistry indexes including cholesterol (CHO), triglyceride (TG), high density lipoprotein-cholesterol (HDL-C), low density lipoprotein-cholesterol (LDL-C) and glucose (Glu) were measured at 0,7 and 12 months of treatment by auto-analyser. 


\section{CONCLUSION}

Chemical and in vitro cell studies have shown preventive properties of these compounds, being a potent antioxidant, against a variety of ROS and RNS. In addition, the epidemiologic and immunopathological studies suggest that consumption of these compounds may lower multiple disease risk. Such potential benefits have been ascribed in part to high concentrations of compounds in nutraceutical treatments. However, these findings have yet only been supported by a small number of intervention trials. By defining the right population and combining antioxidant and immunopathological potentials of these compounds with vitamins and other bioactive plant compounds, the beneficial role of them in other diseases that could be better clarified in future studies.

\section{REFERENCES}

[1]. Luterotti, S., Bicanic, D., Marković, K. \& Franko, M. Carotenes in processed tomato after thermal treatment. Food Control 48, (2015).

[2]. Butler, N. \& Ghugre, P. Effect of beta carotene on the ionisable iron content of wheat. Curr. Res. Nutr. Food Sci. 8, (2020).

[3]. Extraction and Analysis of Beta-carotene Recovery in CPO and Oil Palm Waste by Using HPLC. ARPN J. Eng. Appl. Sci. 11, (2016).

[4]. Qin, Y., Woo, H. J., Shin, K. S., Lim, M. H. \& Lee, S. K. Comparative transcriptome profiling of different tissues from beta-caroteneenhanced transgenic soybean and its nontransgenic counterpart. Plant Cell. Tissue Organ Cult. 140, (2020).

[5]. Druesne-Pecollo, N. et al. Beta-carotene supplementation and cancer risk: A systematic review and metaanalysis of randomized controlled trials. Int. J. Cancer 127, (2010).

[6]. Donhowe, E. G. \& Kong, F. Beta-carotene: Digestion, Microencapsulation, and In Vitro Bioavailability. Food and Bioprocess Technology vol. 7 (2014).

[7]. Avraham, Y. et al. Beta-carotene as a novel therapy for the treatment of "Autistic like behavior" in animal models of Autism. Behav. Brain Res. 364, (2019).

[8]. Huang, J., Weinstein, S. J., Yu, K., Männistö, S. \& Albanes, D. Serum beta carotene and overall and cause-specific mortality: A prospective cohort study. Circ. Res. 123, (2018).

[9]. Li, Q., Sun, Z., Li, J. \& Zhang, Y. Enhancing beta-carotene production in Saccharomyces cerevisiae by metabolic engineering. FEMS Microbiology Letters vol. 345 (2013).

[10]. Bonnefond, $\mathrm{H}$. et al. Coupling and uncoupling of triglyceride and beta-carotene production by Dunaliella salina under nitrogen limitation and starvation. Biotechnol. Biofuels 10, (2017).

[11]. Ding, Y. X. \& Cui, H. Effects of Folic Acid on DNMT1, GAP43, and VEGFR1 in Intrauterine Growth Restriction Filial Rats. Reprod. Sci. 25, (2018).

[12]. Wang, F., Wang, Y., Ma, Q., Cao, Y. \& Yu, B. Development and characterization of folic acidconjugated chitosan nanoparticles for targeted and controlled delivery of gemcitabinein lung cancer therapeutics. Artif. Cells, Nanomedicine Biotechnol. 45, (2017).

[13]. Naim, M. Y. et al. Folic acid enhances early functional recovery in a piglet model of pediatric head injury. Dev. Neurosci. 32, (2011).

[14]. Bwatanglang, I. B. et al. Folic acid targeted Mn:ZnS quantum dots for theranostic applications of cancer cell imaging and therapy. Int. J. Nanomedicine 11, (2016).

[15]. Chalupsky, K., Kračun, D., Kanchev, I., Bertram, K. \& Görlach, A. Folic Acid Promotes 
Recycling of Tetrahydrobiopterin and Protects Against Hypoxia-Induced Pulmonary Hypertension by Recoupling Endothelial Nitric Oxide Synthase. Antioxidants Redox Signal. 23, (2015).

[16]. Fischer, F. et al. Folic acid and creatine improve the firmness of human skin in vivo. J. Cosmet. Dermatol. 10, (2011).

[17]. Yang, S. et al. Non-covalent complexes of folic acid and oleic acid conjugated polyethylenimine: An efficient vehicle for antisense oligonucleotide delivery. Colloids Surfaces B Biointerfaces 135, (2015).

[18]. Rana, S. et al. Folic acid conjugated Fe3O4 magnetic nanoparticles for targeted delivery of doxorubicin. Dalt. Trans. 45, (2016).

[19]. Lv, T. et al. Role of generation on folic acidmodified poly(amidoamine) dendrimers for targeted delivery of baicalin to cancer cells. Mater. Sci. Eng. C 75, (2017).

[20]. Carron, P. M. et al. Targeting the Folate Receptor: Improving Efficacy in Inorganic Medicinal Chemistry. Curr. Med. Chem. 25, (2018).

[21]. Qiu, J. et al. The antitumor effect of folic acid conjugated-Auricularia auricular polysaccharide-cisplatin complex on cervical carcinoma cells in nude mice. Int. J. Biol. Macromol. 107, (2018).

[22]. Wang, X. et al. Preparation of Folic AcidTargeted Temperature-Sensitive Magnetoliposomes and their Antitumor Effects In Vitro and In Vivo. Target. Oncol. 13, (2018).

[23]. Tian, Y. et al. Folic acid-targeted etoposide cubosomes for theranostic application of cancer cell imaging and therapy. Med. Sci. Monit. 23, (2017).

[24]. Kapoor, M. S. et al. Stable Liposome in Cosmetic Platforms for Transdermal Folic acid delivery for fortification and treatment of micronutrient deficiencies. Sci. Rep. 8, (2018).
[25]. Feng, R. et al. Y-shaped Folic Acid-Conjugated PEG-PCL Copolymeric Micelles for Delivery of Curcumin. Anticancer. Agents Med. Chem. 17, (2016).

[26]. Chiani, M. et al. Folic acid conjugated nanoliposomes as promising carriers for targeted delivery of bleomycin. Artif. Cells, Nanomedicine Biotechnol. 46, (2018).

[27]. Stallivieri, A., Baros, F., Jetpisbayeva, G., Myrzakhmetov, B. \& Frochot, C. The Interest of Folic Acid in Targeted Photodynamic Therapy. Curr. Med. Chem. 22, (2015).

[28]. Czeizel, A. E., Dudás, I., Vereczkey, A. \& Bánhidy, F. Folate deficiency and folic acid supplementation: The prevention of neuraltube defects and congenital heart defects. Nutrients vol. 5 (2013).

\section{Cite this article as :}

Bharat Kwatra, Rachit Modi, "Therapeutic Potentials and Applications of Folic Acid and Beta Carotene", International Journal of Scientific Research in Science and Technology (IJSRST), Online ISSN : 2395-602X, Print ISSN : 2395-6011, Volume 7 Issue 4, pp. 271-282, July-August 2020 . Available at doi : https://doi.org/10.32628/IJSRST1207481 Journal URL : http://ijsrst.com/IJSRST1207481 